\title{
Grupo de psicoterapia para pacientes con adicciones hospitalizados en tiempos de la COVID-19
}

\section{Inpatient group psychotherapy for addiction patients in times of COVID-19}

\author{
Magalí Andreu*, María Teresa Pons*, Lourdes Navarro*, Pablo Barrio*. \\ * Addictive Behaviors Unit. Psychiatry Department. Clinical Neuroscience Institute, Clinic Hospital of Barcelona, Barcelona, \\ Spain.
}

\section{La pandemia y la psicoterapia grupal hospitalaria}

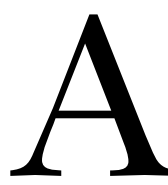

medida que evoluciona la pandemia de la COVID-19, se ha vuelto cada vez más difícil encontrar un área relacionada con la salud que no directa o indirectamente (Legido-Quigley et al., 2020). La psicoterapia grupal para pacientes hospitalizados no ha sido una excepción. Este informe describe el recorrido de nuestro grupo de pacientes con adicciones hospitalizados durante la pandemia.

Un desafío en sí mismo, desde marzo de 2020 la psicoterapia grupal en unidades de hospitalización para pacientes con abuso de sustancias se ha enfrentado a problemas importantes. El primero, común a muchos procedimientos de salud, ha sido su suspensión debida a la escasez de recursos relacionada con la pandemia o al reajuste de prioridades. Además, ha sido cada vez más difícil para los profesionales sanitarios sobrecargados mantener el ritmo con sus tareas rutinarias, en particular cuando las tareas dependen de su propia motivación y perseverancia (Rubino, Luksyte, Perry y Volpone, 2009). Por ejemplo, la psicoterapia grupal en unidades de hospitalización puede ser un procedimiento no retribuido, o en muchas salas puede que ni siquiera se considere una parte esencial de la atención de pacientes con abuso de sustancias (Bandelow et al., 2016; Emond y Rasmussen, 2012).

Segundo, dadas las medidas de aislamiento y distancia social implementadas a nivel mundial, la terapia grupal se ha convertido en un desafío, incluida su versión para pacientes hospitalizados. En Cataluña, por ejemplo, estuvieron prohibidas durante muchos meses las reuniones de más de seis personas. Aunque la telemedicina podría ser una alternativa válida en entornos ambulatorios (Uscher-Pines et al., 2020), los entornos hospitalarios actuales carecen de la posibilidad de incorporar soluciones en línea. Por tanto, deben haberse adaptado totalmente a esta restricción. Eso significa que la selección de pacientes ha sido uno de los grandes desafíos de la psicoterapia grupal en unidades de hospitalización, donde debe haberse implementado una priorización.

Otro obstáculo ha sido la obligación de llevar mascarilla, que, en España, como en muchos otros países, ha sido obligatoria en todo momento. Creemos que este no es un tema menor, ya que los informes describen las implicaciones que 
puede tener el uso de mascarillas en la comunicación interpersonal y en la interpretación de emociones, constructos fundamentales en la psicoterapia grupal (Carbon, 2020).

Por último, además de eso, las adicciones y la salud mental podrían enfrentar un desafío mayor: el de competir con otras áreas sanitarias por la asignación de recursos, un tema importante dada la limitación de los recursos de salud y la crisis económica derivada de esta pandemia ( $\mathrm{Li}$, 2020). Como ha mostrado la historia, el estigma será una barrera significativa en este nuevo capítulo (Vigo, Kestel, Pendakur, Thornicroft y Atun, 2019; Volkow, 2020).

Dicho todo esto, nuestra intención durante la pandemia ha sido abogar por la continuidad de la psicoterapia grupal en unidades de hospitalización para pacientes con abuso de sustancias; creemos que ha sido posible superar muchas de las barreras antes mencionadas y hemos sentido, además, que la necesidad de dar continuación a tal grupo ha sido mayor que nunca.

\section{Aprovechando circunstancias difíciles}

Comenzando con las medidas de seguridad de la COVID-19, el grupo de pacientes hospitalizados en realidad tiene ventajas significativas con respecto a los grupos en entornos ambulatorios. En la actualidad es una práctica común en muchas salas realizar pruebas de detección de SARS-CoV-2 a los pacientes antes de la admisión. Eso, junto con las pruebas frecuentes de los profesionales y el programa de vacunación prácticamente completo de los trabajadores de la salud, hace que el entorno hospitalario sea seguro y libre de SARS-CoV-2.

Aunque en nuestra experiencia el grupo puede funcionar con bastante eficacia a pesar de las mascarillas, si creemos que el paciente y el grupo pueden beneficiarse enormemente de una expresión facial completa, de manera excepcional pedimos a los pacientes que bajen momentáneamente la mascarilla para compartir lo que deben compartir. Curiosamente, a veces sentimos que esto equipara simbólicamente la expresión de la emoción y el compartir a otras actividades vitales, como comer, para las que se puede renunciar al uso de la mascarilla.

Sin embargo, incluso en un entorno seguro, todavía debemos lidiar con las limitaciones legales. Por ejemplo, limitaciones de capacidad. En el caso de Cataluña, el número máximo de personas que pueden reunirse a la misma hora y en el mismo lugar ha sido seis (terapeutas incluidos) durante ocho meses. Eso ha resultado en grupos de cinco pacientes (con un terapeuta) o cuatro pacientes (si también asiste un coterapeuta). Por tanto, la creación de grupos pequeños ha sido la única posibilidad de terapia grupal para pacientes hospitalizados. En nuestra experiencia, también ha sido una oportunidad para un verdadero "grupo pequeño», donde un número reducido de participantes puede desarrollar grupos de mayor intimidad y cohesión. Para que eso suceda, sin embargo, debe haberse implementado una cuidadosa selección de pacientes. De hecho, la selección de pacientes podría considerarse uno de los grandes desafíos de una terapia grupal exitosa (Gans y Counselman, 2010). Dadas las limitaciones legales actuales, también se ha vuelto primordial en la terapia grupal para pacientes hospitalizados.

Operamos en una Unidad de Conductas Adictivas para pacientes hospitalizados de ocho camas, integrada en una Sala de Psiquiatría Aguda de 24 camas. Un psiquiatra, en coterapia con un psicólogo clínico, dirige nuestro grupo de pacientes hospitalizados. Antes de la COVID-19, sin limitaciones de capacidad, solíamos invitar a todos los pacientes de la sala, incluso a aquellos que no estaban bajo el cuidado del equipo de Adicciones. En general, esto resultó en grupos de entre 8 y 12 pacientes. Bajo las restricciones actuales, ha sido obligatorio seleccionar a los pacientes. En ese sentido, hemos cumplido tres directivas principales. Primero, hemos priorizado a los pacientes con abuso de sustancias bajo nuestro propio cuidado. Aunque la terapia «combinada» ha suscitado una gran controversia (Gans, 1990), creemos que, en tales circunstancias, puede mejorar el trabajo terapéutico gracias al conocimiento previo de las ambivalencias, resistencias y necesidades de los pacientes. Segundo, también se ha priorizado a los pacientes con un mayor nivel de funcionamiento y disposición. Por último, hemos tratado de mantener la estabilidad en los pacientes que asisten al grupo para lograr una mayor cohesión. Al hacerlo, esperábamos un mayor beneficio del trabajo grupal para los pacientes. No es sorprendente que nos hayamos encontrado con algunos pacientes que se han sentido decepcionados o excluidos del grupo. En este caso, lo habitual es brindarles una respuesta honesta en su terapia individual con respecto a la imposibilidad de participar en la terapia grupal. En nuestra experiencia, la mayoría de los pacientes ha comprendido fácilmente la situación y se ha tranquilizado tras asegurarles que, a pesar de no poder asistir al grupo, reciben una atención adecuada. En conjunto, abogamos por una selección cuidadosa de los pacientes, un hecho que se ha determinado como crucial para el éxito de la terapia grupal (Kösters, Burlingame, Nachtigall y Strauss, 2006).

Otro tema clave para la supervivencia de la psicoterapia grupal en unidades de hospitalización para pacientes con abuso de sustancias durante esta pandemia ha sido la utilización de los espacios.

La arquitectura de nuestra unidad de hospitalización ofrece dos salas principales para las actividades de los pacientes fuera de sus habitaciones. La sala más grande es donde se sirven las comidas. La segunda sala, significativamente más pequeña, es la ubicación habitual de las actividades grupales y otras actividades terapéuticas. Cabe señalar que nuestra Unidad funciona bajo una política de puertas cerradas. Todas las ventanas están cerradas y los pacientes no tienen acceso al «aire exterior» mientras están en la sala. Durante los dos primeros meses de la pandemia, no se realizó terapia grupal. Luego, cuando los temores iniciales estuvieron bajo control, sentimos la necesidad y la responsabilidad de retomar la terapia grupal. Solicitamos una reunión con los coordinadores médicos y de enfermería. Expusimos nuestras razones y nues- 
tro compromiso con la seguridad de los pacientes y la calidad de la atención, y retomamos nuestra terapia grupal. Desde entonces, cuando la incidencia de la COVID-19 ha vuelto a ser alarmantemente alta, hemos cambiado la ubicación a la sala más grande y con mejor ventilación. Aunque el espacio tiene algunos inconvenientes importantes, como su ubicación en frente de la estación de enfermería y en medio del corredor principal (ambos factores afectan la privacidad del grupo), hemos preferido mantener la terapia grupal en condiciones menos favorables que dejar de hacerla.

\section{La importancia de psicoterapia grupal en unidades de hospitalización}

Más allá de argumentar la factibilidad de soluciones y adaptaciones técnicas, creemos que es básico argumentar a favor de las razones que deben obligar a las salas a continuar su terapia grupal con sus pacientes hospitalizados, a pesar de las dificultades actuales.

Teniendo en cuenta la escasez de recursos sanitarios derivada de la recesión económica relacionada con la pandemia, deben priorizarse los tratamientos rentables (López-Pelayo et al., 2020). La terapia grupal ha demostrado repetidamente serlo (Tucker y Oei, 2007; van der Spek et al., 2018). Vale la pena recordar que, a pesar de la cantidad de evidencia generada sobre que los grupos de pacientes hospitalizados son muy diferentes de las modalidades ambulatorias (Burlingame et al., 2016), también se ha demostrado su eficacia (Kösters et al., 2006).

Además, cabe señalar que la angustia, el aislamiento y la consiguiente soledad se han convertido en características destacadas de esta pandemia (Pfefferbaum y North, 2020). También, en tiempos de crisis, las poblaciones vulnerables, como son las que padecen trastornos por uso de sustancias, suelen sufrir consecuencias desproporcionadas en comparación con la población general. El aislamiento, por ejemplo, es más notorio para los pacientes hospitalizados debido a las restricciones en las visitas de los familiares a los centros sanitarios (Li, 2020). Además, cabe destacar que el personal sanitario también sufre consecuencias de su carga de trabajo excesiva, estrés y agotamiento (Amanullah y Ramesh Shankar, 2020). Esta sobrecarga emocional podría transferirse con facilidad al ambiente de la sala y a los propios pacientes hospitalizados, un hecho bien informado en la literatura (Hall, Johnson, Watt, Tsipa y O'Connor, 2016). Por tanto, afirmamos que no debe excluirse de la estrategia de salud mental que se viene implementando durante estas pandemias la terapia grupal para pacientes hospitalizados con trastornos por uso de sustancias. Los factores terapéuticos en psicoterapia grupal de Yalom pueden mostrarnos fácilmente las razones (Yalom, 1983). La necesidad de infundir esperanza, universalidad, cohesión y catarsis parece casi autoexplicativa para cualquier momento en psiquiatría hospitalaria, pero más aún en el panorama actual.
Una usuaria de cocaína intravenosa con una patología dual de trastorno psicótico se quejaba constantemente de su ingreso obligatorio a la sala de psiquiatría. Informó que la falta de aire fresco y la imposibilidad de caminar fuera de la sala (ambas restricciones debidas a la pandemia) fueron fuentes principales de su angustia durante su ingreso. Sin embargo, asistió de manera continuada a la terapia grupal, en la que pudo hacer un buen trabajo, mostrando una buena comprensión de su adicción a la cocaína. También pudo brindar apoyo de manera coherente a otros pacientes. En su última sesión, antes de ser dada de alta a una sala de largo plazo, se le preguntó sobre su experiencia general en el grupo. Comentó: «Creo que el grupo es lo único realmente positivo que obtuve de mi hospitalización».

\section{Conclusiones}

La COVID-19 probablemente ha sido uno de los mayores cisnes negros para los sistemas de salud modernos, que parecían estar cerca del colapso en algunos momentos durante estos últimos meses. Es probable que la colaboración, la constancia y el compromiso, la motivación y la profesionalidad de los profesionales sanitarios haya evitado mayores daños al sistema.

Aunque lejos de recuperar por completo nuestra normalidad anterior, el avance de las campañas de vacunación en todo el mundo y el descenso de las tasas de incidencia pueden arrojar cierto optimismo a muchos ámbitos, incluso la psicoterapia de grupo. Ya estamos reduciendo la intensidad de algunas de las restricciones que hemos soportado, pero esperamos poder retener muchas de las lecciones que hemos adquirido. Esperamos que las dificultades que ha enfrentado nuestro grupo de pacientes hospitalizados lo hayan fortalecido y preparado mejor para enfrentar los desafíos futuros.

No es un fenómeno nuevo, la crisis siempre trae nuevas oportunidades. Un buen momento para que la psicoterapia grupal hospitalaria demuestre su relevancia.

\section{Conflicto de intereses}

Los autores declaran no tener ningún conflicto de intereses y que no han recibido ningún tipo de financiación para la realización de este artículo.

\section{Referencias}

Amanullah, S. y Ramesh Shankar, R. (2020). The Impact of COVID-19 on physician burnout globally: A review. Healthcare, 8, 421. doi:10.3390/healthcare8040421.

Bandelow, B., Lueken, U., Wolff, J., Godemann, F., Wolff-Menzler, C., Deckert, J.,... Berger, M. (2016). Leitliniengerechte stationäre psychiatrisch-psychotherapeutische/psychosomatische behandlung von angsterkrankungen: Wieviel personal ist erforderlich? 
[Guideline-oriented inpatient psychiatric psychotherapeutic/psychosomatic treatment of anxiety disorders: How many personnel are need?]. Der Nervenarzt, 87, 302-310. doi:10.1007/s00115-016-0085-1.

Burlingame, G. M., Seebeck, J. D., Janis, R. A., Whitcomb, K. E., Barkowski, S., Rosendahl, J. y Strauss, B. (2016). Outcome differences between individual and group formats when identical and nonidentical treatments, patients, and doses are compared: A 25-year meta-analytic perspective. Psychotherapy, 53, 446-461. doi:10.1037/ pst0000090.

Carbon, C. C. (2020). Wearing face masks strongly confuses counterparts in reading emotions. Frontiers in psychology, 11, 566886. doi:10.3389/fpsyg.2020.566886.

Emond, S. y Rasmussen, B. (2012). The status of psychiatric inpatient group therapy: Past, present, and future. Social Work with Groups, 35, 6891. doi:10.1080/01609513.2011 .553711

Gans, J. S. (1990). Broaching and exploring the question of combined group and individual therapy. International journal of group psychotherapy, 40, 123-137. doi:10.1080/0 0207284.1990.11490595.

Gans, J. S. y Counselman, E. F. (2010). Patient selection for psychodynamic group psychotherapy: Practical and dynamic considerations. International journal of group psychotherapy, 60, 197-220. doi:10.1521/ijgp.2010.60.2.197.

Hall, L. H., Johnson, J., Watt, I., Tsipa, A. y O'Connor, D. B. (2016). Healthcare staff wellbeing, burnout, and patient safety: A systematic review. PloS one, 11. doi:10.1371/ journal.pone.0159015.

Kösters, M., Burlingame, G. M., Nachtigall, C. y Strauss, B. (2006). A meta-analytic review of the effectiveness of inpatient group psychotherapy. Group Dynamics, 10, 146163. doi:10.1037/1089-2699.10.2.146.

Legido-Quigley, H., Mateos-García, J. T., Campos, V. R., Gea-Sánchez, M., Muntaner, C. y McKee, M. (2020). The resilience of the Spanish health system against the COVID-19 pandemic. The Lancet. Public health, 5, 251-252. doi:10.1016/S2468-2667(20)30060-8.

Li, L. (2020). Challenges and priorities in responding to COVID-19 in inpatient psychiatry. Psychiatric Services, 71, 624-626. doi:10.1176/appi.ps.202000166.

López-Pelayo, H., Aubin, H. J., Drummond, C., Dom, G., Pascual, F., Rehm, J.,... Gual, A. (2020). "The post-COVID era": Challenges in the treatment of substance use disorder (SUD) after the pandemic. BMC medicine, 18, 241. doi:10.1186/s12916-020-01693-9.

Pfefferbaum, B. y North, C. S. (2020). Mental Health and the Covid-19 pandemic. New England Journal of Medicine, 383, 510-512. doi:10.1056/nejmp2008017.

Rubino, C., Luksyte, A., Perry, S. J. y Volpone, S. D. (2009). How do stressors lead to burnout? The mediating role of motivation. Journal of Occupational Health Psychology, 14, 289-304. doi:10.1037/a0015284.
Tucker, M. y Oei, T. P. S. (2007). Is group more cost effective than individual cognitive behaviour therapy? The evidence is not solid yet. Behavioural and Cognitive Psychotherapy, 35, 77-91. doi:10.1017/S1352465806003134.

Uscher-Pines, L., Sousa, J., Raja, P., Mehrotra, A., Barnett, M. L. y Huskamp, H. A. (2020). Suddenly becoming a "virtual doctor": Experiences of psychiatrists transitioning to telemedicine during the COVID-19 pandemic. Psychiatric services, 71, 1143-1150. doi:10.1176/ appi.ps.202000250.

van der Spek, N., Jansen, F., Holtmaat, K., Vos, J., Breitbart, W., van Uden-Kraan, C. F.,... Verdonck-de Leeuw, I. M. (2018). Cost-utility analysis of meaning-centered group psychotherapy for cancer survivors. Psycho-oncology, 27, 1772-1779. doi:10.1002/pon.4726.

Vigo, D. V., Kestel, D., Pendakur, K., Thornicroft, G. y Atun, R. (2019). Disease burden and government spending on mental, neurological, and substance use disorders, and self-harm: Cross-sectional, ecological study of health system response in the Americas. The Lancet. Public health, 4, 89-96. doi:10.1016/S2468-2667(18)30203-2.

Volkow, N. D. (2020). Stigma and the toll of addiction. New England Journal of Medicine, 382, 1289-1290. doi:10.1056/ nejmp1917360.

Yalom, I. D. (1983). Inpatient Group Psychotherapy. Basic Books. 